\title{
Complete Two Loop Bosonic Contributions to the Muon Lifetime in the Standard Model
}

\author{
M. Awramik ${ }^{1,2}$ and M. Czakon ${ }^{3,2}$ \\ ${ }^{1}$ Institut für Theoretische Teilchenhysik, Universität Karlsruhe, D-76128 Karlsruhe, Germany \\ ${ }^{2}$ Department of Field Theory and Particle Physics, Insitute of Physics, \\ University of Silesia, Uniwersytecka 4, PL-40007 Katowice, Poland \\ ${ }^{3}$ Institut für Theoretische Physik, Universität Karlsruhe, D-76128 Karlsruhe, Germany
}

\begin{abstract}
The last missing correction to the muon lifetime in the Standard Model at $\mathcal{O}\left(\alpha^{2}\right)$ coming from gauge and Higgs boson loops is presented. The associated contribution to the parameter $\Delta r$ in the on-shell scheme ranges from $6 \times 10^{-5}$ to $-4 \times 10^{-5}$ for Higgs boson masses from $100 \mathrm{GeV}$ to $1 \mathrm{TeV}$. This result translates into a shift of the $W$ boson mass which does not exceed $\pm 1 \mathrm{MeV}$ in the same range and amounts in particular to approximately $-0.8 \mathrm{MeV}$ for a $115 \mathrm{GeV}$ Higgs boson.
\end{abstract}

PACS numbers: 12.15.Lk, 13.35.Bv, 14.60.Ef

The muon decay lifetime $\left(\tau_{\mu}\right)$ has long been used as an input parameter for high precision predictions of the Standard Model (SM). It allows for an indirect determination of the mass of the $W$ boson $\left(M_{W}\right)$, which suffers currently from a large experimental error of $39 \mathrm{MeV}$ [1], one order of magnitude worse than that of the $Z$ boson mass $\left(M_{Z}\right)$. A reduction of this error by the Large Hadron Collider (LHC) to $15 \mathrm{MeV}$ [2] and by a future linear collider to $6 \mathrm{MeV}$ [3] would provide a stringent test of the SM by confronting the theoretical prediction with the experimental value.

The extraction of $M_{W}$ with an accuracy matching that of next experiments, i.e. at the level of a few MeV necessitates radiative corrections beyond one loop order. Large two loop contributions from fermionic loops have been calculated in 1 . The current prediction is affected by two types of uncertainties. First, apart from the still unknown Higgs boson mass, two input parameters introduce large errors. The current knowledge of the top quark mass results in an error of about $30 \mathrm{MeV}$ [5], which should be reduced by LHC to $10 \mathrm{MeV}$ and by a linear collider even down to $1.2 \mathrm{MeV}$. The inaccuracy of the knowledge of the running of the fine structure constant up to the $M_{Z}$ scale, $\Delta \alpha\left(M_{Z}\right)$, introduces a further 6.5 MeV error. Second, several higher order corrections are unknown, of which only one at the $\mathcal{O}\left(\alpha^{2}\right)$ order. This lacking contribution comes from diagrams with no closed fermion loops. It has been previously estimated to be of the order of the square of the one loop bosonic correction [ [1]. This, however, is unjustified since both have a different dependence on the Higgs boson mass (logarithmic vs. quadratic). In this Letter, the question of the exact size of this contribution is finally settled.

The muon decay is naturally described in the language of effective field theory. The process' dynamics are driven by the leptons, the five light quarks, and the two massless gauge bosons. Even then the momentum scale set by the muon mass leads to a strong decoupling of the $\tau$ lepton and of the heavier of the five quarks [6]. The heavy particles $W, Z$, the Higgs boson and the top quark gen- erate point interactions. A general effective lagrangian assumes the form

$$
\mathcal{L}_{\text {eff }}=\mathcal{L}_{\mathrm{QED}}+\mathcal{L}_{\mathrm{QCD}}^{(5)}+\sum_{n, i} \frac{\mathcal{C}_{n}^{i}}{\left(M_{W}^{2}\right)^{n}} \mathcal{O}_{n}^{i}
$$

where $\mathcal{L}_{Q E D}$ and $\mathcal{L}_{Q C D}^{(5)}$ are the bare QED and the five flavour QCD lagrangians, $\mathcal{O}_{n}^{i}$ are composite operators of the light fields and $\mathcal{C}_{n}^{i}$ are the respective dimensionless matching coefficients, obtained by comparing the Green functions of the full theory with those of the effective one. The mass of the $W$ boson has been chosen as the heavy scale.

Due to the left-handed nature of the charged current in the SM, the only operator of dimension six relevant to muon decay is the four-fermion interaction of the Fermi model

$$
\mathcal{O}_{F}=\bar{e} \gamma^{\alpha}\left(1-\gamma_{5}\right) \mu \otimes \overline{\nu_{\mu}} \gamma_{\alpha}\left(1-\gamma_{5}\right) \nu_{e}
$$

with $\mu, e, \nu_{\mu}, \nu_{e}$ denoting the spinors of the muon, electron and their neutrinos. Here the so called chargeconserving form has been used (Fierz transformed SM amplitude). The respective matching coefficient is traditionally parametrised as

$$
\mathcal{C}_{F}=M_{W}^{2} \frac{G_{F}}{\sqrt{2}}=\frac{\pi \alpha}{2 s_{W}^{2}}(1+\Delta r),
$$

where $G_{F}$ is known as the Fermi constant, whereas $\Delta r$ represents the higher order corrections and vanishes at the Born level. The one loop corrections in the effective theory given by Eqs. 1, 2 2 and 3 have been calculated in [7] and [8], while the two loop in [9].

The framework for construction of effective lagrangians together with a proof has been given in [10]. It is based on expansions of individual Feynman diagrams. The method is presented on the example of a specific graph contributing to bosonic corrections to muon decay at two loop order in Fig. 1. The terms in the large mass expansion can be classified according to the scale of the loop 
a)

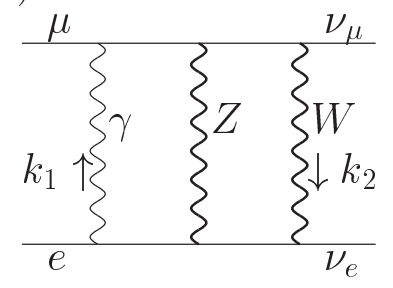

c)

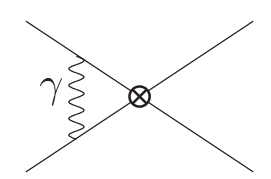

d)

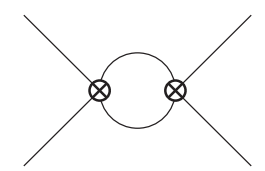

e)

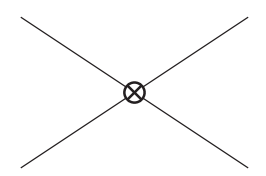

FIG. 1: A typical muon decay diagram a) and the contributions to its large mass expansion according to the momenta b) $k_{1}$-soft, $k_{2}$-soft; c) soft-hard; d) hard-soft; e) hard-hard.

momenta. When both momenta are "soft" $\left(\ll M_{W}\right)$, as in b), the propagators of the $W$ and $Z$ bosons are expanded leading to a correction of order $\alpha / M_{W}^{4}$ in the effective theory. For one momentum "soft" and one "hard" $\left(\sim M_{W}\right)$, as in c) and d), corrections of either order, $\alpha / M_{W}^{2}$ or $1 / M_{W}^{4}$ in the effective theory, are generated. The contribution to the matching coefficient comes only from the region where both momenta are hard, as in e). In this case all of the light particle masses and momenta should be put to zero. By these arguments it can be shown that $\Delta r$ can be obtained by simply taking the sum of all the diagrams and putting all external momenta and light masses to zero. The procedure should generate no spurious infrared divergences, while the physical divergences connected with the photon should be contained in the corrections of the effective theory. As known the Fermi theory corrections are finite, therefore the $\Delta r$ correction obtained as above should also be finite.

Previous calculations of $\Delta r$ have been based on a different method of factorisation originally devised in [11. This procedure consists of subtracting from the infrared divergent SM diagrams the respective Fermi theory diagrams in Pauli-Villars regularisation. The difference is well defined in the limit of zero light masses and external momenta. It turns out, however, that the QED Ward identity, which is responsible for the finiteness of the corrections in the Fermi theory, implies in this case the vanishing of the sum of the subtracted diagrams. This proves that both procedures are equivalent.

The evaluation of two loop corrections to a fourfermion process requires the full second order renormalisation of the SM lagrangian in all but the Higgs sector, where first order suffices. The comparison with experiment imposes the use of on-shell parameters for the final result. Throughout this work the on-shell scheme was

used, with a procedure similar to the one described in [5]. The only substantial difference concerns the treatment of tadpoles.

It is known that gauge invariance of mass counterterms requires inclusion of tadpoles [12, 13] (at the two loop level this has been explicitely shown in 14]). In this case, however, one cannot use one-particle-irreducible (1PI) Green functions. In order to have gauge invariant counter-terms and 1PI Green functions only, a special procedure was designed. An additional renormalisation constant for the bare vacuum expectation value $v_{0}$, denoted $Z_{v}$, has been introduced and explicitely split from the bare masses

$$
\begin{aligned}
v_{0} & \longrightarrow v_{0} Z_{v}^{1 / 2} \\
M_{W, Z}^{0} & \longrightarrow M_{W, Z}^{0} Z_{v}^{1 / 2} .
\end{aligned}
$$

The term linear in the Higgs field $H$ in the lagrangian

$$
T^{0} H^{0}=\frac{M_{W}^{0} s_{W}^{0}}{e_{0}}\left(M_{H}^{0}\right)^{2} Z_{v}^{1 / 2}\left(Z_{v}-1\right) H^{0},
$$

is then used to determine $Z_{v}$, through the requirement that tadpoles are canceled. It can be proved [12, 15] that the bare masses are gauge invariant in this case (an equivalent procedure which makes use of the effective potential has been used in [16]).

The calculation of the two loop bosonic contributions to muon decay was performed by means of a completely automated system. The diagram generation stage was done by the $\mathrm{C}++$ library DiaGen [17]. The tensor reduction of two loop propagator diagrams was accomplished with the algorithm described in [18], whereas vacuum diagrams were treated with integration by parts identities [19]. For algebraic manipulations, the program FORM 20] was used. The two loop two-point integrals were numerically evaluated with single integral representations of the package S2LSE [21]. The latter was modified for quadruple precision, which was needed due to large cancellations (independent terms grow as $M_{H}^{8}$, while the result behaves as $M_{H}^{2}$ ).

The size of the software required several tests. The following algebraic checks were performed

- ultraviolet and infrared finiteness, by cancellation of poles in dimensional regularisation,

- gauge invariance, by independence of the three gauge parameters of the general $R_{\xi}$ gauge for the SM,

- Slavnov-Taylor identities for two-point functions, as given in [18], both for on-shell integrals and by expansion in the external momentum to second order.

Several numerical tests were also done 


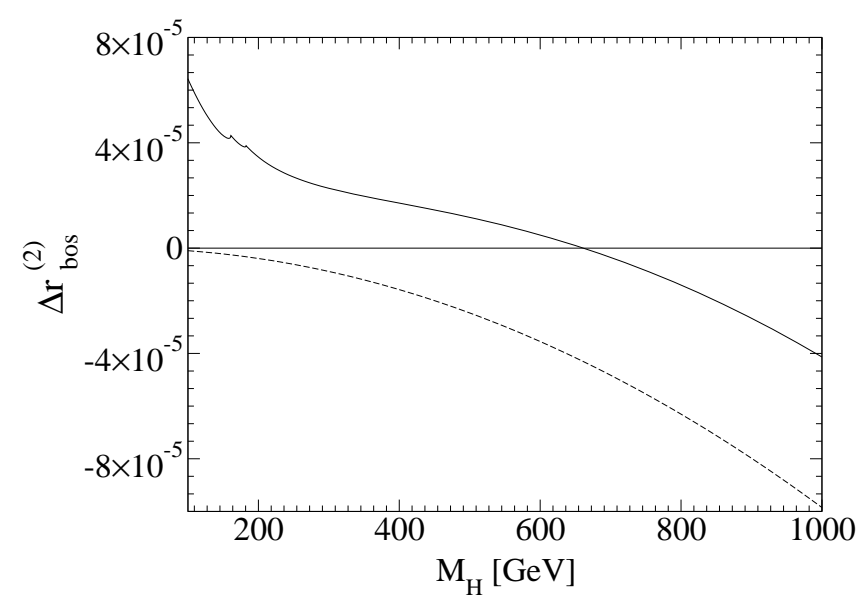

FIG. 2: $\Delta r$ as function of the Higgs boson mass (solid line) and the leading term in the large Higgs mass expansion (dashed line).

- all of the master integrals were evaluated independently by means of deep mass difference and large mass expansions,

- each of the two-point on-shell diagrams was calculated separately with the help of small-momentum and different large-mass expansions,

- the result of 14 for the $W$ and $Z$ mass counterterms was reproduced to precision dictated by the order of the expansions contained therein.

A detailed description of the methods used is relegated to a subsequent publication [15].

The variation of the bosonic contribution with $M_{H}$ subtracted at $M_{H}=100 \mathrm{GeV}$ has also been compared with the one presented in [5]. A discrepancy which grows up to $\sim 16 \%$ for $M_{H}=1 \mathrm{TeV}$, has been found.

All the numerical values have been obtained for the following coupling and mass parameters [1]

$$
\begin{aligned}
& \alpha^{-1}=137.03599976(50) \\
& G_{F}=1.16639(1) \times 10^{-5} \mathrm{GeV}^{-2} \\
& M_{W}=80.423(39) \mathrm{GeV} \\
& M_{Z}=91.1876(21) \mathrm{GeV}
\end{aligned}
$$

The two loop bosonic correction to $\Delta r$, denoted $\Delta r_{\text {bos }}^{(2)}$, is shown in Fig. 2 as function of the Higgs boson mass $M_{H}$. Its behaviour is very smooth apart from the range between $150 \mathrm{GeV}$ and $190 \mathrm{GeV}$, where two peaks are located. Their positions at $\sim 161 \mathrm{GeV}$ and $\sim 182 \mathrm{GeV}$ correspond to the $W$ and $Z$ pair production thresholds introduced through the on-shell definition of the Higgs boson mass.

It is interesting to study the correction for large values of $M_{H}$. In fact, two different leading $M_{H}^{2}$ terms have been given in 22 and 23], both based on the same results

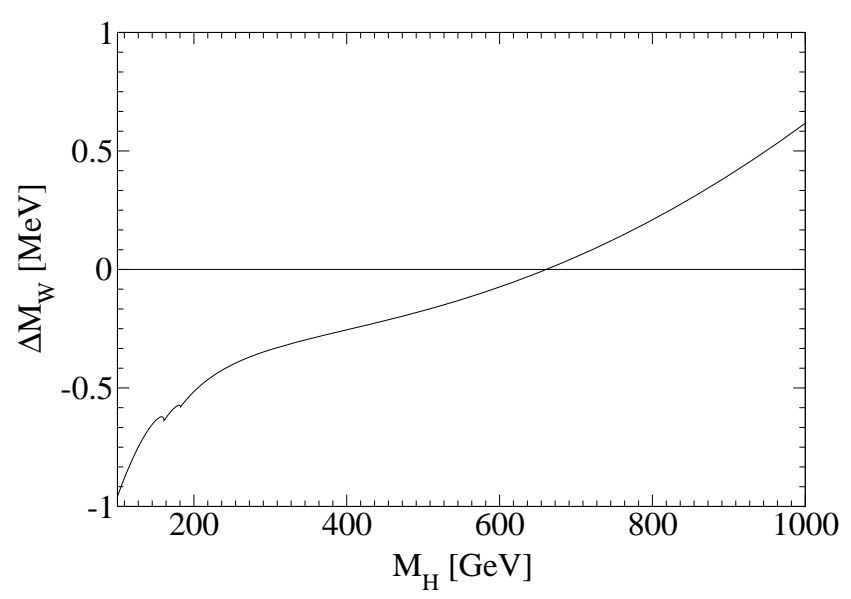

FIG. 3: $W$ boson mass shift generated by two loop bosonic corrections.

of 24]. In this last work a renormalisation scheme has been used in which $M_{H}^{2}$ terms occur only in two-point functions. The leading behaviour can then be obtained from the formula

$\Delta r_{\text {Higgs }}^{(2)}=2 \frac{\delta e}{e}+\frac{\Delta M_{W}^{2}}{M_{W}^{2}}+\frac{c_{W}^{2}}{s_{W}^{2}}\left(\frac{\Delta M_{Z}^{2}}{M_{Z}^{2}}-\frac{\Delta M_{W}^{2}}{M_{W}^{2}}-\Delta \rho\right)$,

where $\delta e$ is the charge renormalisation and $\Delta M_{W, Z}$ represent the shift of the $W$ and $Z$ self energies from zero external momentum up to the on-shell value. $\Delta \rho$ has been introduced in 25]. With the help of [24], the result reads

$$
\begin{aligned}
\Delta r_{\text {Higgs }}^{(2)} & =\left(\frac{\alpha}{4 \pi s_{W}^{2}}\right)^{2} \frac{M_{H}^{2}}{8 M_{W}^{2}} \\
& \times\left(9 \sqrt{3} \mathrm{Cl}_{2}\left(\frac{\pi}{3}\right)+\frac{49}{72}-\frac{11 \pi \sqrt{3}}{4}-\frac{25 \pi^{2}}{108}\right)
\end{aligned}
$$

where $\mathrm{Cl}_{2}$ is the Clausen function and $\mathrm{Cl}_{2}(\pi / 3)=$ 1.0149416. Eq. 9 is in agreement with [22, whereas in 23. the charge renormalisation has not been included. The leading Higgs mass dependence is depicted in Fig. 2. The difference between the full result and Eq. 9 for values below $1 \mathrm{TeV}$ shows the importance of subleading corrections. It has been checked that for $M_{H}>5 \mathrm{TeV}$ both functions agree very well.

With the help of $\Delta r$ the $W$ boson mass can be estimated by inverting Eq. 3

$$
M_{W}=M_{Z} \sqrt{\frac{1}{2}+\sqrt{\frac{1}{4}-\frac{\pi \alpha}{\sqrt{2} G_{F} M_{Z}^{2}}(1+\Delta r)}} .
$$

This equation should be solved recursively, since $\Delta r$ also depends on $M_{W}$. In the case of small corrections one can, however, obtain the additional mass shift by means 
of an expansion. For parameters' values given in Eq. 17, a second order Taylor series yields

$$
\Delta M_{W}=-(1.491+1.779 \overline{\Delta r}) \times 10^{4} \Delta r_{\text {bos }}^{(2)}[\mathrm{MeV}]
$$

where $\overline{\Delta r}$ represents the dominating correction, which is composed of one loop electroweak, two loop fermionic and $\mathcal{O}\left(\alpha \alpha_{S}\right)$ QCD contributions. Since $\overline{\Delta r}$ does not exceed $5 \%$ [5], the third order in the Taylor series would contribute less than a percent which is comparable to the inaccuracy induced by the dependence of $\Delta r_{b o s}^{(2)}$ on the $W$ mass.

The $W$ mass shift, Eq. 11, with $\overline{\Delta r}$ neglected, is plotted in Fig. 3. The dropped term implies an error of less than $6 \%$, which is, however, completely negligible in view of the size of the total effect, which does never exceed $\pm 1 \mathrm{MeV}$.

In conclusion, the complete $\mathcal{O}\left(\alpha^{2}\right)$ bosonic contributions to muon decay have been calculated in the on-shell scheme, and shown to lead to a small $W$ boson mass shift below $1 \mathrm{MeV}$. A framework for evaluation of $\Delta r$ which does not explicitely refer to the QED corrections in the Fermi theory has been presented and its equivalence to the method based on subtraction of Pauli-Villars regulated diagrams demonstrated. A procedure of obtaining gauge invariant mass counter-terms without explicitely including tadpole graphs has been developed.

The authors would like to thank K. Chetyrkin, O. Veretin and A. Onishchenko for fruitful discussions and A. Freitas for providing the results of [5]. M. A. would like to thank the "Marie Curie Programme" of the European Commission for a stipend. M. C. would like to thank the Alexander von Humboldt foundation for fellowship. This work was supported in part by the European Community's Human Potential Programme under contract HPRN-CT-2000-00149 Physics at Colliders, and by the KBN Grants 5P03B09320 and 2P03B05418.

[1] K. Hagiwara et al. [Particle Data Group Collaboration], Phys. Rev. D 66, 010001 (2002).

[2] ATLAS Collaboration, CERN/LHCC/99-15 (1999); CMS Collaboration, CMS TDR 1-5 (1997/98);
S. Haywood et al., hep-ph/0003275.

[3] TESLA Technical Design Report, Part III, eds. R. Heuer, D. J. Miller, F. Richard and P. M. Zerwas, DESY-200111C, hep-ph/0106315;

T. Abe et al., hep-ex/0106057.

[4] G. Degrassi, P. Gambino and A. Vicini, Phys. Lett. B 383, 219 (1996);

G. Degrassi, P. Gambino and A. Sirlin, Phys. Lett. B 394, 188 (1997);

A. Freitas, W. Hollik, W. Walter and G. Weiglein, Phys. Lett. B 495, 338 (2000)

[5] A. Freitas, W. Hollik, W. Walter and G. Weiglein, Nucl. Phys. B 632, 189 (2002)

[6] T. van Ritbergen and R. G. Stuart, Phys. Lett. B 437, 201 (1998)

[7] S. M. Berman, Phys. Rev. 112, 267 (1958).

[8] T. Kinoshita and A. Sirlin, Phys. Rev. 113, 1652 (1959).

[9] T. van Ritbergen and R. G. Stuart, Phys. Rev. Lett. 82, 488 (1999)

[10] S. G. Gorishnii, Nucl. Phys. B 319, 633 (1989).

[11] A. Sirlin, Phys. Rev. D 22, 971 (1980).

[12] T. Appelquist, J. Carazzone, T. Goldman and H. R. Quinn, Phys. Rev. D 8, 1747 (1973);

[13] J. C. Taylor, Gauge Theories Of Weak Interactions, (Cambridge University Press, Cambridge, 1976);

J. Fleischer and F. Jegerlehner, Phys. Rev. D 23, 2001 (1981).

[14] F. Jegerlehner, M. Y. Kalmykov and O. Veretin, Nucl. Phys. B 641, 285 (2002).

[15] M. Awramik, M. Czakon, A. Onishchenko and O. Veretin, hep-ph/0209084.

[16] A. I. Bochkarev and R. S. Willey, hep-ph/9607420;

R. S. Willey, Phys. Rev. D 64, 034014 (2001).

[17] M. Czakon, in preparation.

[18] G. Weiglein, R. Scharf and M. Bohm, Nucl. Phys. B 416, 606 (1994)

[19] K. G. Chetyrkin and F. V. Tkachov, Nucl. Phys. B 192, 159 (1981).

[20] J. A. Vermaseren, math-ph/0010025.

[21] S. Bauberger, F. A. Berends, M. Bohm and M. Buza, Nucl. Phys. B 434, 383 (1995)

[22] F. Halzen, B. A. Kniehl and M. L. Stong, Z. Phys. C 58, 119 (1993).

[23] F. Jegerlehner, Prog. Part. Nucl. Phys. 27, 1 (1991).

[24] J. van der Bij and M. J. Veltman, Nucl. Phys. B 231, 205 (1984);

J. J. van der Bij, Nucl. Phys. B 248, 141 (1984).

[25] D. A. Ross and M. J. Veltman, Nucl. Phys. B 95, 135 (1975). 